\title{
Selecting and optimal sizing of hybridized energy storage systems for tidal energy integration into power grid
}

\author{
Seifeddine BEN ELGHALI ${ }^{1}$, Rachid OUTBIB ${ }^{1}$, Mohamed BENBOUZID ${ }^{2,3}$ (I)
}

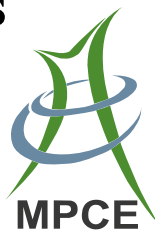

\begin{abstract}
The high penetration of renewable energy systems with fluctuating power generation into the electric grids affects considerably the electric power quality and supply reliability. Therefore, energy storage resources are used to deal with the challenges imposed by power variability and demand-supply balance. The main focus of this paper is to investigate the appropriate storage technologies and the capacity needed for a successful tidal power integration. Therefore, a simplified sizing method, integrating an energy management strategy, is proposed. This method allows the selection of the adequate storage technologies and determines the required least-cost storage capacity by considering their technological limits associated with different power dynamics. The optimal solutions given by the multi-objective evolutionary algorithm are presented and analyzed.
\end{abstract}

CrossCheck date: 21 June 2018

Received: 30 July 2017 / Accepted: 21 June 2018 / Published online: 3 September 2018

(C) The Author(s) 2018

$\square$ Mohamed BENBOUZID

Mohamed.Benbouzid@univ-brest.fr

Seifeddine BEN ELGHALI

Seifeddine.Benelghali@lis-lab.fr

Rachid OUTBIB

Rachid.Outbib@lis-lab.fr

1 LIS-UMR CNRS 7020, University of Aix-Marseille, Marseille, France

2 UMR CNRS 6027 IRDL, University of Brest, Brest, France

3 Shanghai Maritime University, Shanghai, China
Keywords Tidal energy, Energy storage system, Optimal sizing, Selection

\section{Introduction}

The integration of renewable energies into the electrical grid is one of the most challenging tasks. In fact, the quality of the power delivered to the grid becomes very crucial when the penetration level of renewable energies is very high $[1,2]$. Therefore, the use of energy storage systems (ESSs) can alleviate potential problems. ESS can provide a variety of application solutions along the entire electricity system value chain, from generation support to transmission and distribution support to end-customer uses [3]. Consequently, different ESS applications have been defined and analyzed according to their uses and value of benefits.

For renewable applications, it is common to use ESS for energy time-shift and capacity firming. The energy time shift increases the value of energy and so profits are increased. Indeed, most renewable energy resources produce a significant portion of electric energy at off-peak periods which has a low financial value. As a result, ESS can be charged and used when demand is high and supply is tight $[4,5]$. By contrast, capacity firming allows the use of intermittent electric supply as a nearly constant source. Such use may reduce power-related charges and/or offset the need for equipment. Likewise, for effective renewable integration, some requirements are identified and classified in two categories. The first one is the short duration applications including the reduction of power volatility and the improvement of power quality. The second one concerns the long duration applications embracing the reduction of output variability, the transmission congestion 
relief, the back-up for unexpected power generation shortfalls and the minimization of load violations.

Satisfying all the earlier announced requirements makes the sizing task very complicated and depending on many parameters (e.g., resource variability, load fluctuation, technologies limitations, life time, costs, etc.). In this context, too many papers in literature deal with the optimal sizing of energy storage systems especially for renewable energy applications [6-10]. The ESS sizing problem was mainly studied either in the time domain [11-13] or in the frequency domain [14-16]. Moreover, it can be noticed that the common practice was the use of one or two preselected ESS and then try to find its optimal sizes according to some defined objectives [17-19].

In contrast, this paper proposes a new approach allowing the selection of the adequate storage technology and determines the required least-cost storage capacity by considering its technological limits associated to different power dynamics. Therefore, a simplified sizing method, integrating an energy management strategy, is proposed. To highlight its effectiveness, the proposed strategy is applied to a tidal energy system, but it can be employed with any other renewable energy such as photovoltaic (PV), wind turbine, etc. This paper is organized as follows. First, Section 2 recalls the particularities of the tidal energy and describes the power fluctuation dynamics. Subsequently, Section 3 announces the adopted energy management strategy. The approach to the selection of the appropriate ESS is defined in Section 4. Section 5 presents the sizing optimization algorithm. Lastly, Section 6 concludes the paper and provides directions for future research.

\section{Power fluctuation dynamics}

In order to model the whole system, multi-physics approach was adopted including the resource, the marine turbine, and the ESS. This simulator can evaluate marine current turbine performances and dynamic loads over different operating conditions. Throughout the paper, we will use $\mathcal{P}=\left(T_{0}, T_{P}\right)$, with $0 \leq T_{0}<T_{P}$, to denote the period of analysis.

The kinetic power harnessed by a marine current turbine (MCT) can be calculated as:

$$
P_{M C T}(t)=\frac{1}{2} \rho C_{p} A V_{\text {tide }}^{3}(t) \quad t \in \mathcal{P}
$$

where $V_{\text {tide }}$ is the the current speed in the turbine cross section; $\rho$ is the sea water density; $C_{p}$ is the power coefficient; $A$ represents the swept rotor area.

Equation (1) expresses that the power produced by the MCT is proportional to the cube of the current speed in the turbine cross section.For more accuracy, the swell effect, which is considered as the most disturbing one for the considered resource model, is added based on Stokes model. This model is a very classical first-order model used to predict the swell influence in the sea water column. For a given swell amplitude $H$, a period $T$, a swell length $L$ and ground sea depth $d$, the speed potential $\phi$ can be calculated for each depth $z$. The water speed created by the swell effect can be deduced by a spatial derivation of this potential [20].

$$
\left\{\begin{array}{l}
V_{\text {swell }}=\operatorname{grad} \phi \\
\phi=-\frac{H L}{T} \frac{\cosh 2 \pi\left(\frac{z+d}{L}\right)}{\sinh 2 \pi\left(\frac{d}{L}\right)} \sin 2 \pi\left(\frac{t}{T}-\frac{x}{L}\right)
\end{array}\right.
$$

This speed disturbance, calculated for typical intense swell specifications, can be added to the predicted tidal current speed to estimate how the swell can disturb the tidal current values in the turbine disk.

Thus, the $P_{M C T}$ is highly dependent on the fluctuations in the marine current speed [21-23]. Two main kinds of power fluctuations can be identified: on a large time scale the generated power fluctuates over a period of 6 or 12 hours which is related to tidal astronomical phenomena; on a small time scale it can fluctuate with a period of a few seconds to several minutes shown in Fig. 1. Consequently, these fluctuations affect the power storage system which can be evaluated by:

$$
P_{s s}(t)=P_{M C T}(t)-P_{\text {grid }}(t) \quad t \in \mathcal{P}
$$

where $P_{\text {grid }}$ is the targeted power to be delivered to the grid. More precisely, $P_{\text {grid }}$ can be any continuous function in time and having values in $\mathbb{P}=\left(P_{\text {grid }}^{\min }, P_{\text {grid }}^{\max }\right)$. This function can be chosen to answer to some specific uses. However, in this work, and for sake of simplicity, this function is assumed to be constant on $\mathcal{P}$.

From (3), $P_{s s}$ is defined positive during the charging period and negative during the discharge period. In order to highlight the different dynamics of the storage power flow, a fast fourier transform (FFT) is established. Figure 2 shows three scales of dynamics which obviously need different types of storage system.

\section{Energy management strategy based on a frequency approach}

According to the FFT, a hybridization of three types of storage systems corresponding to the different dynamics of power flow seems to be the best solution to deliver smooth power to the grid. In order to determine the part of each 


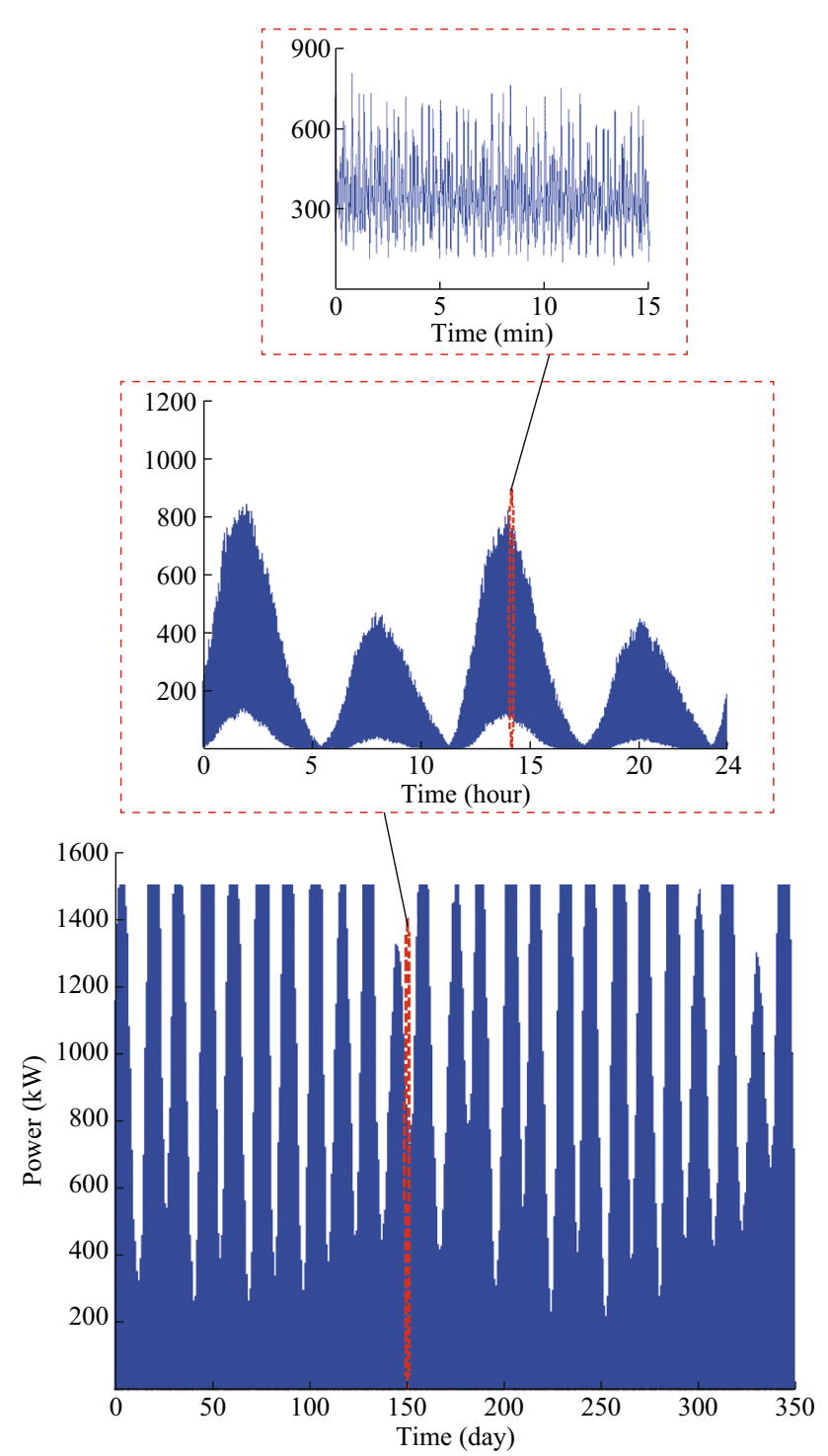

Fig. 1 Harnessed tidal power during one year

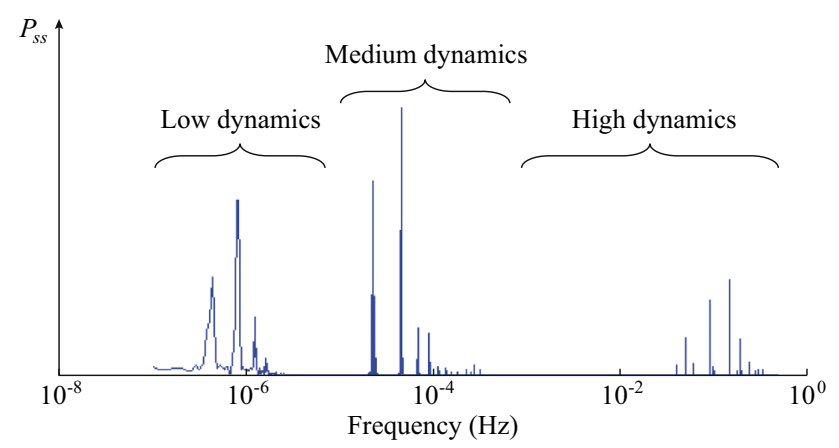

Fig. 2 FFT of power storage system

ESS, an energy management based on frequency approach is tested [24, 25]. Two low pass filters, defined in (4), are used to share out the power flow $P_{s s}$ between the 3 ESSs

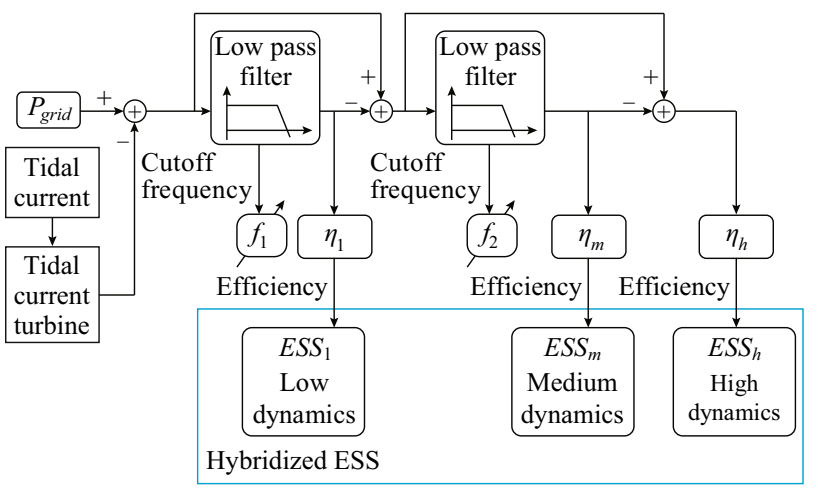

Fig. 3 Energy management procedure

shown in Fig. 3. $E S S_{l}, E S S_{m}, E S S_{h}$ are devoted to storage low, medium and high dynamics, respectively.

$$
\left\{\begin{array}{l}
P_{E S S_{l}}(s)=\eta_{l}\left(\frac{2 \pi f_{1}}{2 \pi f_{1}+s}\right) P_{s s}(s) \\
P_{E S S_{m}}(s)=\eta_{m}\left(\frac{2 \pi f_{2}}{2 \pi f_{2}+s}\right)\left(P_{s s}(s)-P_{E S S_{l}}(s)\right) \\
P_{E S S_{h}}(s)=\eta_{h}\left(P_{s s}(s)-P_{E S S_{l}}(s)-P_{E S S_{m}}(s)\right)
\end{array}\right.
$$

with $\eta$ is the ESS efficiency defined by:

$\eta_{k}=\left\{\begin{array}{ll}\frac{1}{\eta_{E S S}} & P_{E S S} \geq 0 \\ \eta_{E S S} & P_{E S S}<0\end{array} \quad k \in\{l, m, h\}\right.$

In this paper, the ESS efficiency is considered equal during the charge and discharge operations shown in Table 1.

This approach ensures the compatibility between the frequency components of the power flow and the intrinsic characteristics of the different sources.

\section{Selection of ESSs}

Storage system performance characteristics for any power applications can be described in terms of two parameters, i.e., specific power and specific energy. Figure 4 shows different types of ESSs in the energy-power plane called "Ragone chart" and includes information about the suitable application time period for each element [26].

As it can be noticed, batteries are more suitable for applications with long term variations on the scale of minutes to several hours, while superconducting magnetic energy storage systems and ultra-capacitors are more adapted for applications on the time scale of several seconds.

Accordingly, the two key criteria to consider when selecting an energy storage system are the system power and the energy ratings. Based on the energy management 
Table 1 Technical and economical characteristics of electrical energy storage technologies

\begin{tabular}{llllll}
\hline Technologies & $\begin{array}{l}\text { Energy density }(\mathrm{J} / \\
\mathrm{L})\end{array}$ & $\begin{array}{l}\text { Power density } \\
(\mathrm{W} / \mathrm{L})\end{array}$ & $\begin{array}{l}\text { Power capital cost }(\$ / \\
\mathrm{kW})\end{array}$ & $\begin{array}{l}\text { Energy capital cost }(\$ / \\
\mathrm{kWh})\end{array}$ & $\begin{array}{l}\text { Charge/discharge efficiency } \\
(\%)\end{array}$ \\
\hline PHS & $210^{3}-5.510^{3}$ & $0.5-1.5$ & $2500-4300$ & $5-100$ & 87 \\
CAES & $210^{3}-710^{3}$ & $3-6$ & $400-1000$ & $2-120$ & $70-79$ \\
Flywheel & $3.610^{6}-1810^{6}$ & $20-80$ & $250-350$ & $1000-5000$ & $90-93$ \\
Lead $\{-\}$ acid & $3610^{3}-144010^{3}$ & $50-80$ & $300-600$ & $200-400$ & 85 \\
Li\{-\}ion & $5.410^{6}-3610^{6}$ & $200-500$ & $1200-4000$ & $600-2500$ & 85 \\
NaS & $50010^{3}-65010^{3}$ & $150-300$ & $1000-3000$ & $300-500$ & 85 \\
NiCd & $29010^{3}-210^{6}$ & $15-150$ & $500-1500$ & $800-1500$ & 85 \\
VRFB & $210^{3}-710^{3}$ & $16-33$ & $600-1500$ & $150-1000$ & $75-82$ \\
ZnBr & $3.610^{3}-9010^{3}$ & $30-60$ & $700-2500$ & $150-1000$ & $60-70$ \\
Capacitor & $36010^{6}+$ & $2-10$ & $200-400$ & $500-1000$ & $75-90$ \\
Double-layer & $36010^{6}+$ & $10-30$ & $100-300$ & $300-2000$ & $95-98$ \\
$\quad$ capacitor & $3.610^{6}-1410^{6}$ & $0.2-6.1$ & $200-300$ & $1000-10000$ & 95 \\
SMES & $210^{6}-3.610^{6}$ & $500-3000$ & $300-1500$ & $2-15$ & 59 \\
Hydrogen fuel cell & & & & \\
\hline
\end{tabular}

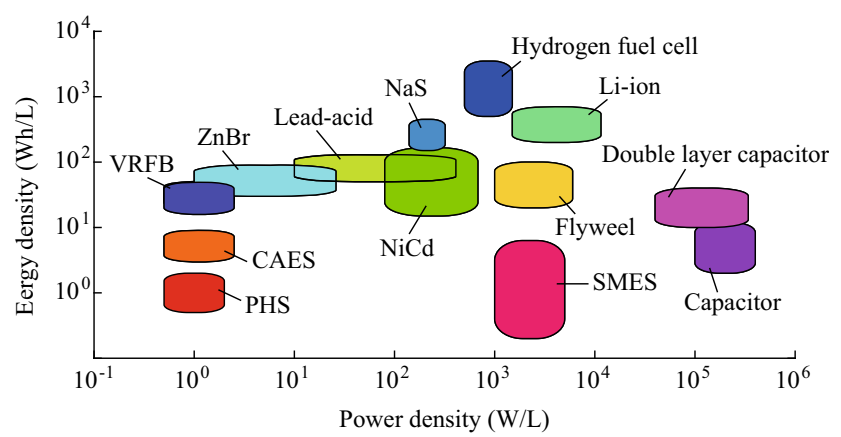

Fig. 4 Ragone chart

strategy, $P_{E S S}^{\max }$ the power upper limit that an ESS cannot exceed when supplying energy and $E_{E S S}^{A}$ the active energy required to efficiently smooth the delivered power are estimated for each ESS. The evolution of the ESS energy is given by:

$E_{E S S}(t)=\int_{T_{0}}^{t} P_{E S S}(\tau) \mathrm{d} \tau \quad t \in \mathcal{P}$

Thus, the stored active energy $E_{E S S}^{A}$ can be expressed by:

$E_{E S S}^{A}=\max _{t \in \mathcal{P}}\left(E_{E S S}(t)\right)-\min _{t \in \mathcal{P}}\left(E_{E S S}(t)\right)$

The maximum power of the ESS is defined as:

$P_{E S S}^{\max }=\max _{t \in \mathcal{P}}\left(\left|P_{E S S}(t)\right|\right)$

Figures 5, 6, 7 and 8 show an example of power and energy variations within 24 hours for $E S S_{m}$ within 15 minutes for $E S S_{m}$ obtained through the energy management strategy. In

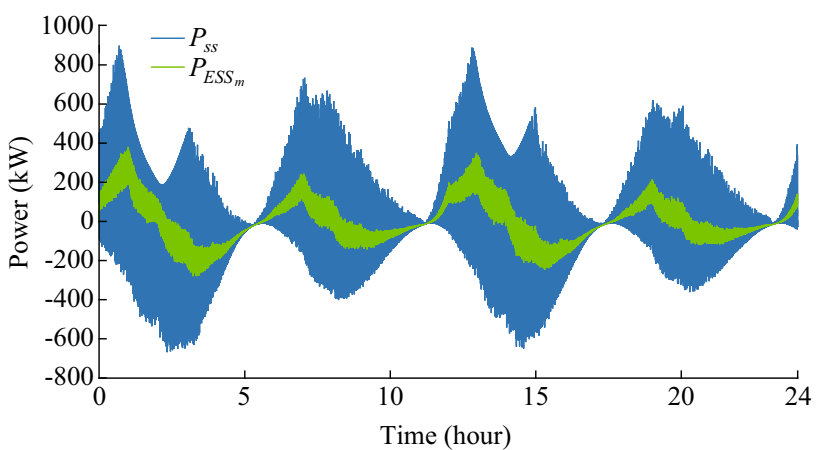

Fig. $5 E S_{m}$ power fluctuation over 24 hours

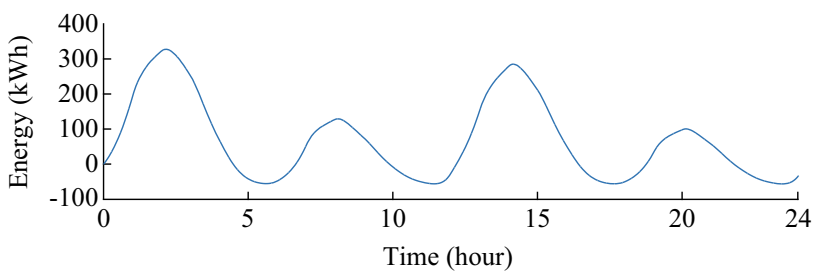

Fig. $6 E S S_{m}$ energy fluctuation over 24 hours

order to create a relation between the power flow dynamics and the different storage system technologies, the notion of specific frequency [27] is introduced and defined as the ratio between the power density $\rho_{E S S}^{P}$ and the energy density $\rho_{E S S}^{E}$ : 


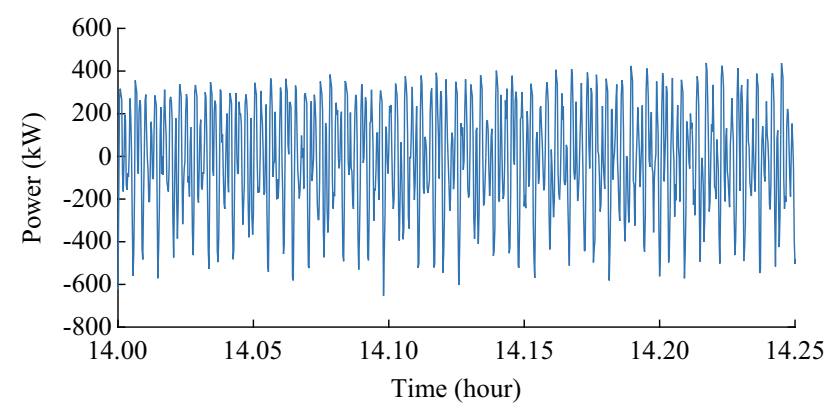

Fig. $7 \mathrm{ESS}_{h}$ power fluctuation over 15 minutes

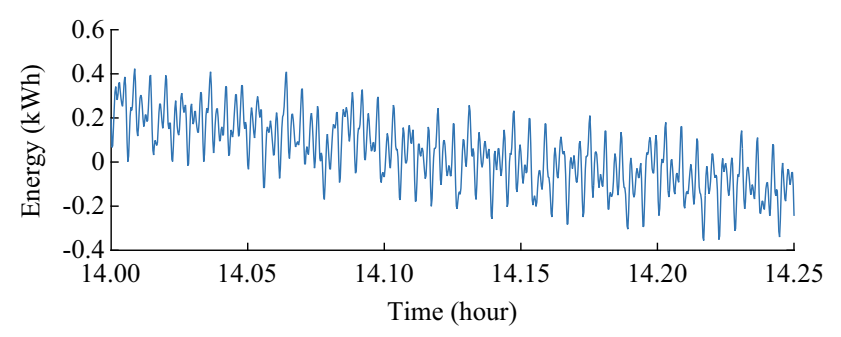

Fig. $8 E S_{h}$ energy fluctuation over 15 minutes

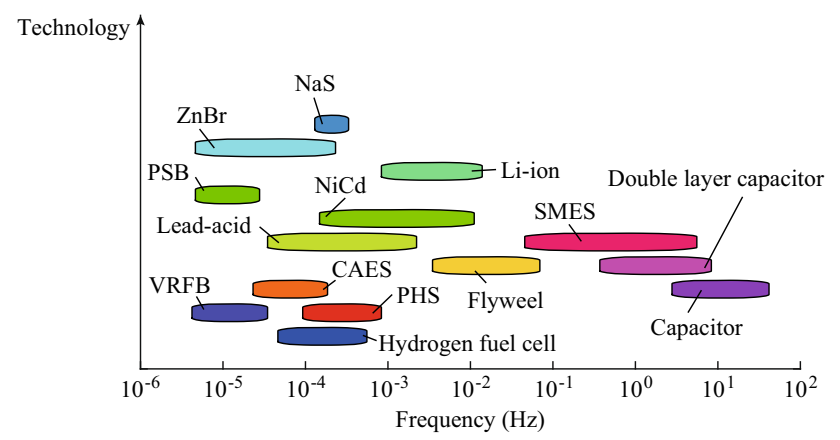

Fig. 9 Power Ragone chart in frequency plane

$f[\mathrm{~Hz}]=\frac{\rho_{E S S}^{P}[\mathrm{~W} / \mathrm{L}]}{\rho_{E S S}^{E}[\mathrm{~J} / \mathrm{L}]}$

Therefore, the different elements of the Ragone chart can be reported on frequency plane using (9) as shown in Fig. 9. The storage system specifications, summarized in Table 1, are based on data collected from [28]. Similarly, the specific frequency of the installed ESS can be defined as:

$f_{E S S}=\frac{P_{E S S}^{\max }}{E_{E S S}^{A}}$

\section{Sizing optimization}

\subsection{Problem formulation}

In this work, the objective of the sizing optimization consists in maximizing the energy delivered to the grid during a period $\mathcal{P}$ with a minimum of power fluctuations and using the least cost solution. It is assumed that over the period $\mathcal{P}, P_{M C T}$ is known by using prediction model based on the predictability of tidal coefficients. In our study, $P_{M C T}$ is calculated over one year using a turbulent resource [29]. Let $P_{\text {grid }}^{\text {real }}$ be the real power delivered to the grid expressed by:

$P_{\text {grid }}^{\text {real }}(t)=P_{M C T}(t)+\sum_{k \in \mathcal{D}} \alpha_{k} P_{E S S_{k}}(t) \quad t \in \mathcal{P}$

where $\mathcal{D}=\{l, m, h\} ; \alpha_{k}(k \in \mathcal{D})$ is set equal to one when $E S S_{k}$ is selected and equal to zero elsewhere. Let also $E_{\text {grid }}^{\text {real }}$ denote the real energy delivered to the grid expressed by:

$E_{\text {grid }}^{\text {real }}(t)=\int_{T_{0}}^{t} P_{\text {grid }}^{\text {real }}(\tau) \mathrm{d} \tau \quad t \in \mathcal{P}$

Let also $\Delta P_{\text {grid }}^{\text {real }}$ be the power variation expressed by:

$\Delta P_{\text {grid }}^{\text {real }}=\max _{t \in \mathcal{P}} P_{\text {grid }}^{\text {real }}(t)-\min _{t \in \mathcal{P}} P_{\text {grid }}^{\text {real }}(t)$

For now on, we use $C_{E S S_{k}}(k \in \mathcal{D})$ to design the cost of $E S S_{k}$. We assume that $C_{E S S_{k}} \in \mathcal{S}_{E S S_{k}}(k \in \mathcal{D})$ where $\mathcal{S}_{E S S_{k}}$ is the set of costs of the selected solutions for each dynamic (low, medium and high). In order to estimate the cost of each storage system, the volume needed for a given $\operatorname{ESS}_{k}(k \in \mathcal{D})$ is expressed by:

$$
V_{k}=\max \left(\frac{E_{E S S_{k}}^{T o t}}{\rho_{E S S_{k}}^{E}}, \frac{P_{E S S_{k}}^{\max }}{\rho_{E S S_{k}}^{P}}\right)
$$

with

$E_{E S S_{k}}^{T o t}=\frac{E_{E S S_{k}}^{A}}{D O D_{E S S_{k}}}$

where $D O D_{E S S_{k}}$ is the depth of discharge. If the volume is obtained from the energy ratio, the system cost will be calculated as:

$C_{E S S_{k}}=C_{E S S_{k}}^{E c} E_{E S S_{k}}^{T o t} \quad k \in \mathcal{D}$

where $C_{E S S_{k}}^{E c}$ is the energy capital cost $(\$ / \mathrm{kWh})$. In the case where the volume is sized by the power ratio, the cost will be given by:

$C_{E S S_{k}}=C_{E S S_{k}}^{P c} P_{E S S_{k}}^{\max }$

where $C_{E S S_{k}}^{P c}$ denotes the power capital cost $(\$ / \mathrm{kW})$. For reasons of simplification, only average values of capital costs, calculated from Table 1, are considered. In order to be more realistic, it should be noted that operating and maintenance costs must be considered.

In this study, the sizing variables considered are the power grid $P_{\text {grid }}$ and the two cut-off frequencies $f_{1} \in \mathcal{F}_{1}$ 


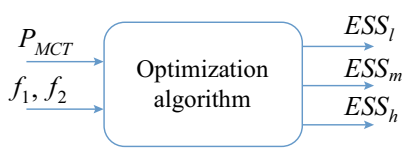

Fig. 10 Problem of sizing optimization

and $f_{2} \in \mathcal{F}_{2}$ shown in Fig. 10 where $\mathcal{F}_{1}$ and $\mathcal{F}_{2}$ denote the sets of all admissible frequencies.

Now, we are in position to state the problem formulation. By using the notations introduced above, the problem of sizing optimization aims to solve simultaneously three sub-problems that are:

$$
\begin{aligned}
O F_{E}= & \max _{P_{\text {grid }} \in \mathbb{P}} E_{\text {grid }}^{\text {real }}\left(P_{\text {grid }}\right) \quad(S P 1) \\
O F_{\Delta P}= & \min _{f_{1} \in \mathcal{F}_{1}} \Delta P_{\text {grid }}^{\text {real }}\left(f_{1}, f_{2}, P_{\text {grid }}\right) \\
& f_{2} \in \mathcal{F}_{2} \\
& P_{\text {grid }} \in \mathbb{P}
\end{aligned}
$$

and

$$
\begin{aligned}
O F_{C}= & \min _{f_{1} \in \mathcal{F}_{1}} C_{E S S}\left(f_{1}, f_{2}, P_{\text {grid }}\right) \\
& f_{2} \in \mathcal{F}_{2} \\
& P_{\text {grid }} \in \mathbb{P}
\end{aligned}
$$

with

$C_{E S S}=\sum_{k \in \mathcal{D}} \alpha_{k} C_{E S S_{k}}$

The first objective function is $O F_{E}$ aims to maximize the annual energy delivered to the grid, the second one $O F_{\Delta P}$ intends to minimize the power fluctuation while the third function $O F_{C}$ targets the total cost minimization of the selected storage systems. However, and generally speaking, the three sub-problems do not possess necessarily a common solution. Thus, we rather seek to solve the problem of sizing optimization can be formulated as:

$$
\begin{aligned}
O F_{G}= & \min _{f_{1} \in \mathcal{F}_{1}} F\left(E_{\text {grid }}^{\text {real }}, \Delta P_{\text {grid }}^{\text {real }}, C_{E S S}\right) \\
& f_{2} \in \mathcal{F}_{2} \\
& P_{\text {grid }} \in \mathbb{P}
\end{aligned}
$$

where $F$ is a suitable function expressing a global objective.

Besides, the problem (17) must be solved by respecting the following constraints:

$$
P_{\text {grid }}^{\text {real }}(t) \in\left(P_{\text {grid,min }}^{\text {real }}(t), P_{\text {grid,max }}^{\text {real }}(t)\right) \quad t \in \mathcal{P}
$$

where

$$
\left\{\begin{array}{l}
P_{\text {grid,min }}^{\text {real }}(t)=P_{M C T}(t)-P_{s s}(t)-\Delta P_{\text {grid }}^{\text {real }} \\
P_{\text {grid,max }}^{\text {real }}(t)=P_{M C T}(t)-P_{s s}(t)+\Delta P_{\text {grid }}^{\text {real }}
\end{array}\right.
$$

with, and by using the third equality of (3):

$P_{s s}(t)=\sum_{k \in \mathcal{D}} P_{E S S_{k}}(t)$

It should be noted that the optimization problem stated by (17) is complex and in our knowledge it can not be solved explicitly. Besides, the number of possibility, depending on desired sampling accuracy and the technology types, can become very large. Hence, one must seek for a suitable strategy that solve the problem by respecting a balance between the accuracy and the computation time.

\subsection{Strategy for solving problem}

In this work, the problem is solved by using the multiobjective optimization based on the non-dominated sorting

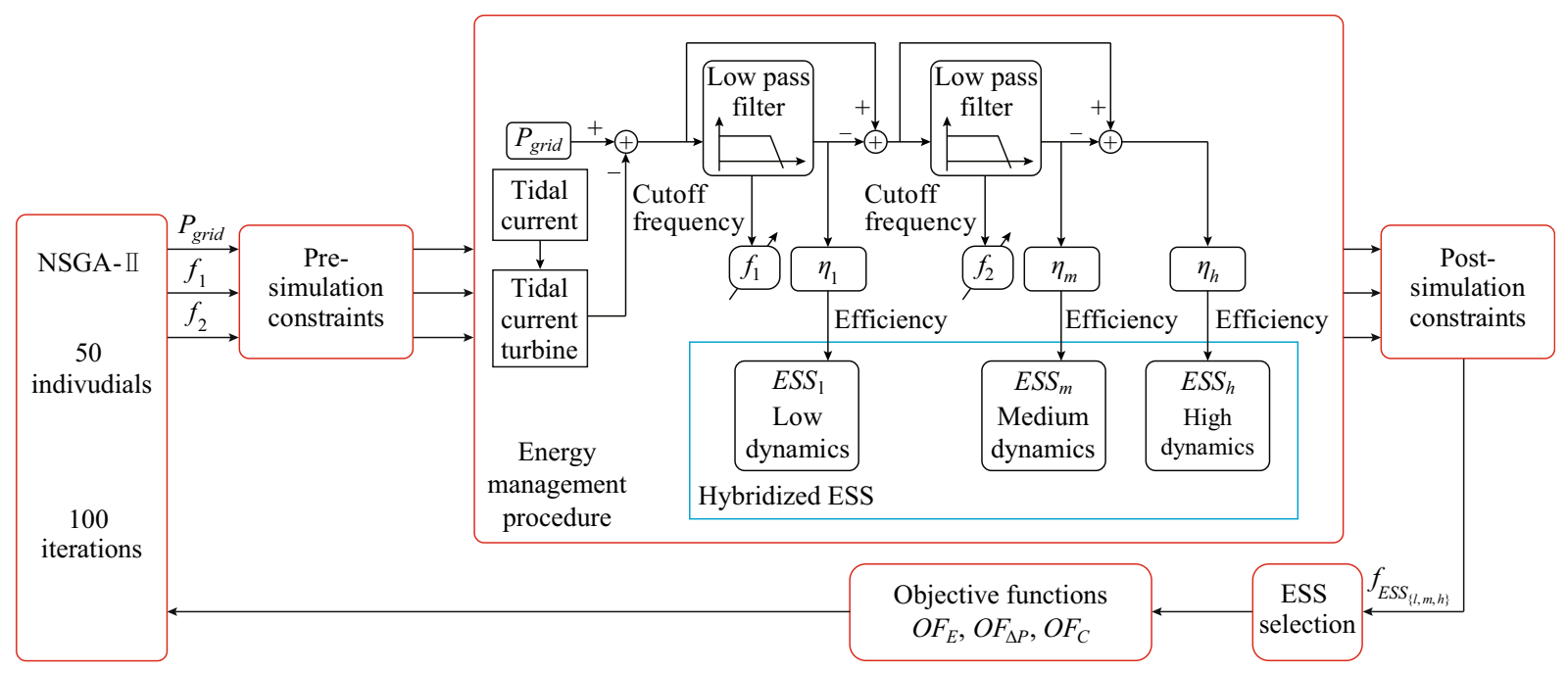

Fig. 11 Optimization process 
genetic algorithm-II (NSGA-II). Indeed, NSGA-II, in most problems, is able to find much better spread of solutions and better convergence near the true Pareto-optimal front compared to Pareto-archived evolution strategy and strength-Pareto evolutionary algorithm [30]. The optimization strategy is described by Fig. 11 and is given by Algorithm 1.

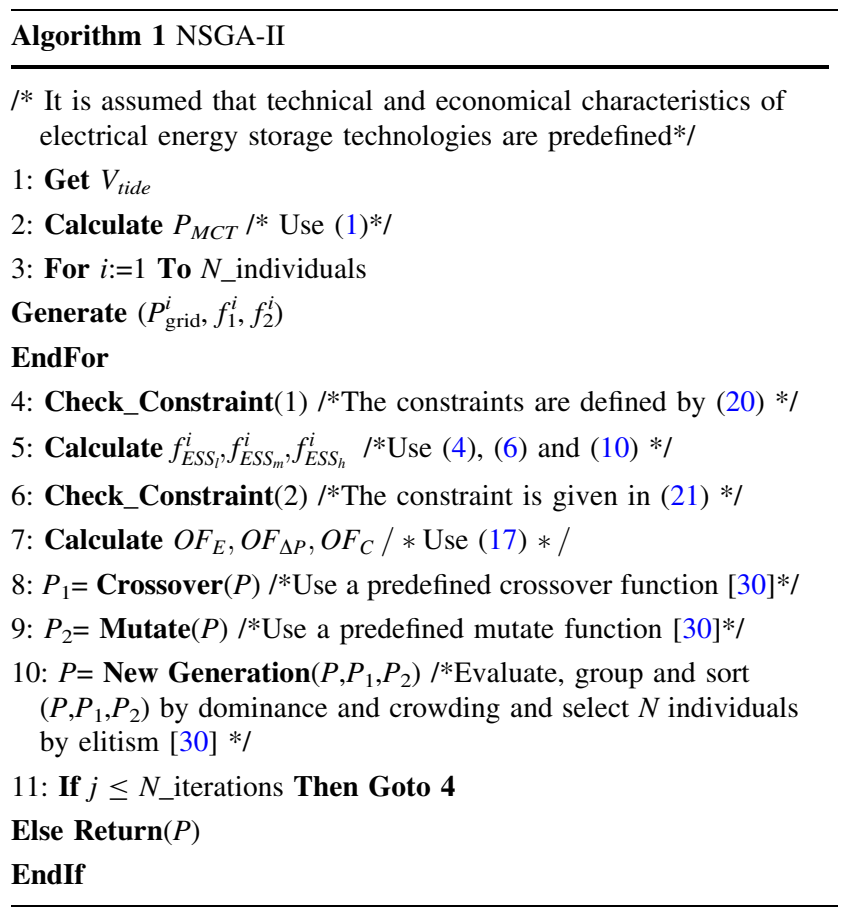

The pre-simulation constraints considered in this study are:

$\left\{\begin{array}{l}f_{1} \in \mathcal{F}_{1}=\left(f_{1}^{\text {min }}, f_{1}^{\text {max }}\right) \\ f_{2} \in \mathcal{F}_{2}=\left(f_{2}^{\text {min }}, f_{2}^{\text {max }}\right) \\ P_{\text {grid }} \in \mathbb{P}\end{array}\right.$

with

$0 \leq P_{\text {grid }}^{\min }<P_{\text {grid }}^{\max } \leq P_{M C T}^{\max }$

The post-simulation constraints are:

$f_{E S S} \in\left(f_{E S S}^{\min }, f_{E S S}^{\max }\right)$

\subsection{Results and discussion}

The non-dominated sorting genetic algorithm NSGA-II is applied to the optimization process [30]. The numerical values used for the analysis are given in Table 2.

The best trade-offs are projected in the $3 \mathrm{D}$ plane $\left(O F_{E}, O F_{\Delta P}, O F_{C}\right)$ showin in Fig. 12. The best optimal solution is given for the individual minimizing the three
Table 2 Numerical values used for analysis and simulation

\begin{tabular}{ll}
\hline Constant & Value \\
\hline$C_{p}$ & 0.4 \\
$P_{M C T}$ & $1500 \mathrm{~kW}$ \\
$N_{\text {_individuals }}$ & 50 \\
$N_{-}$iterations & 100 \\
$\rho$ & $1000 \mathrm{~kg} / \mathrm{m}^{3}$ \\
$f_{1}^{\min }$ & $5 \times 10^{-6} \mathrm{~Hz}$ \\
$f_{2}^{\min }$ & $5 \times 10^{-5} \mathrm{~Hz}$ \\
$f_{1}^{\max }$ & $5 \times 10^{-5} \mathrm{~Hz}$ \\
$f_{2}^{\max }$ & $5 \times 10^{-2} \mathrm{~Hz}$ \\
$P_{\text {grid }}^{\min }$ & $100 \mathrm{~kW}$ \\
$P_{\text {grid }}^{\max }$ & $1 \mathrm{MW}$ \\
$\left(P_{\text {grid }}^{\text {real }}\right)_{\text {avg }}$ & $210 \mathrm{~kW}$ \\
\hline
\end{tabular}

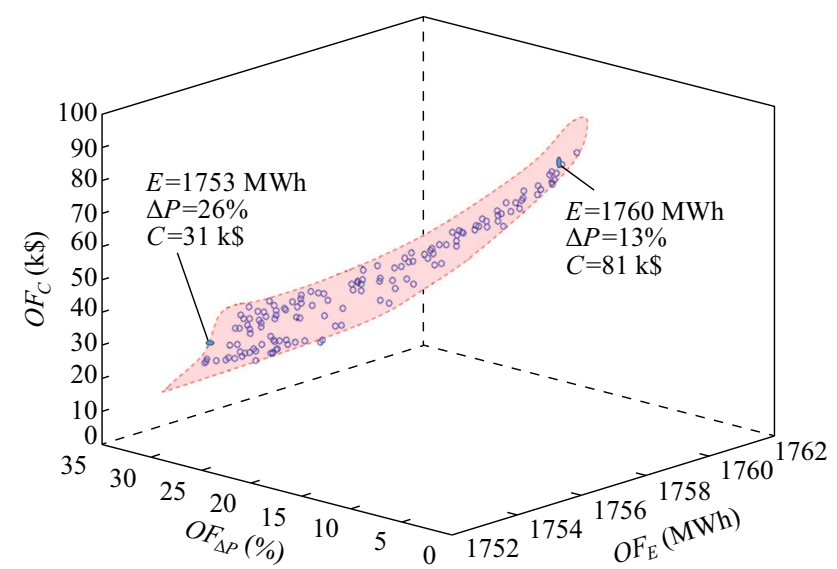

Fig. 12 Projection of the Optimal Pareto front onto $\left(O F_{E}, O F_{\Delta P}, O F_{C}\right)$ plane

objective functions. However, as it can be noticed, there is no absolute minimum solution. Therefore, a compromised solution can be obtained by giving more importance to two of the three criteria. In our case, we focused on the total cost and the power fluctuation shown in Fig. 13. This choice is justified by the fact that the annual energy does not change too much according the $O F_{E}$ axis.

Figure 14 shows the energy storage system selected for the two points shown in Fig. 12. The first solution is obtained with the hybridization of double layer capacitor (DLC) and hydrogen fuel cell systems with a total cost of $81 \mathrm{k} \$$ and only $13 \%$ of power variation. While the second solution is based on the combination of the SMES and hydrogen fuel cell systems with a total cost of $31 \mathrm{k} \$$ and only $26 \%$ of power variation. In both cases, the low dynamic presented by the frequency $f_{E S S_{1}}$ does not correspond to any storage system, therefore only two storage 


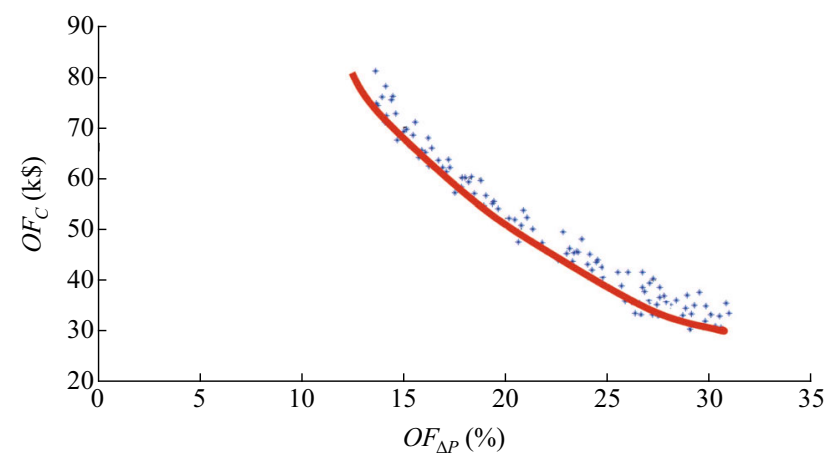

Fig. 13 Projection of the Optimal Pareto front onto $\left(O F_{\Delta P}, O F_{C}\right)$ plane

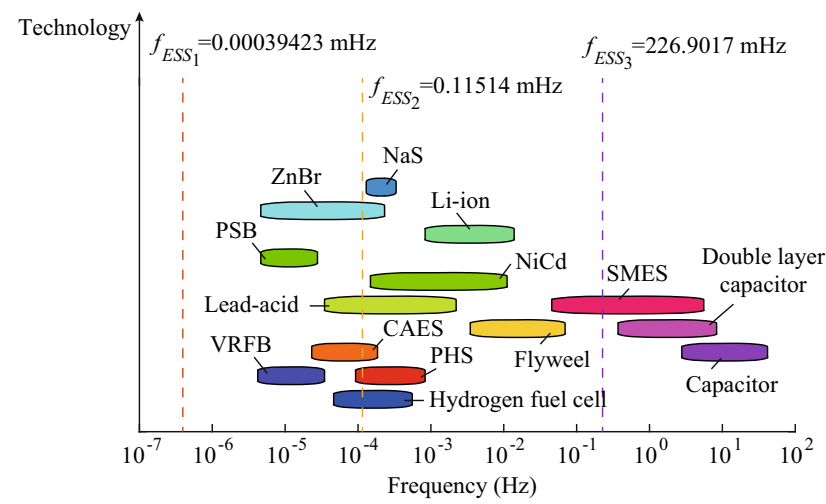

Fig. 14 Example of solution based on hydrogen fuel cell and SMES

systems are selected. Thus, the real power delivered to the grid will be smoothed according to high and medium dynamics and will present a low dynamic fluctuation shown in Fig. 15. For better smoothing performances, other types of storage systems may be used but it will leads to very expensive solutions. Indeed, a more smoothed power $P_{\text {grid }}^{\text {real }}$ was expected since we were using a constant $P_{\text {grid }}$ value as input for the NSGA-II algorithm. Therefore, we investigated other solutions from second and third Pareto-front rank. Figure 16 shows an hybridized solution where three types of ESS are selected (VRFB, PHS and SMES). In this particular case, the ESS cost is ten times higher than the DLC and hydrogen system with practically no power variation shown in Fig. 17). Thus, despite of its good performances according to the second objective function $O F_{\Delta P}$, the solution is sorted at the second Paretofront due to its very high cost.

The obtained results highlights the fact that only the medium and high dynamic must be considered for the ESS sizing problem in tidal energy applications. Indeed, the astronomic nature of the tidal energy resource makes it predictable for low dynamics. Therefore by filtering the power generated by the daily moon cycle, swell effect and

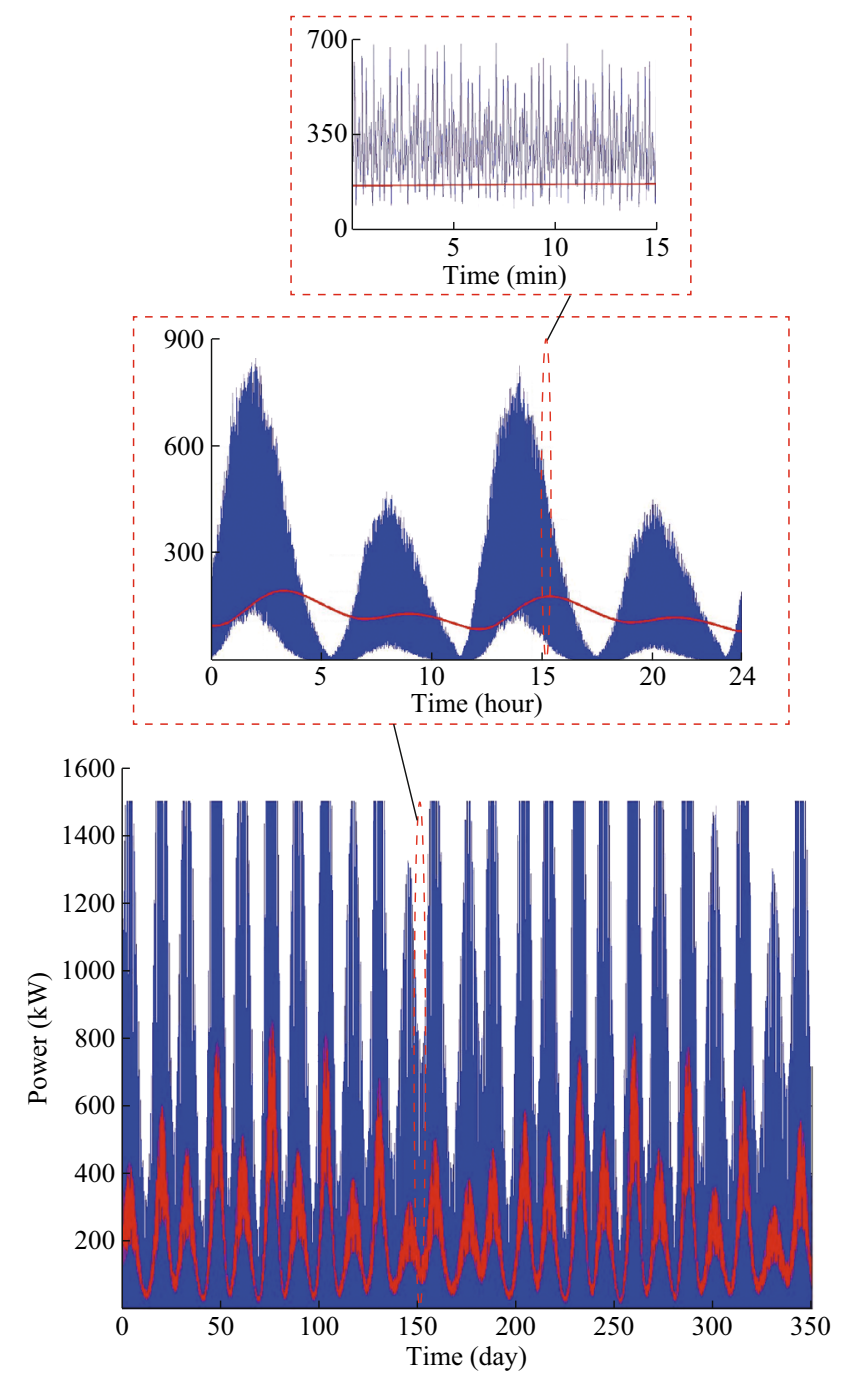

Fig. 15 Example of filtered power grid from the first set solutions

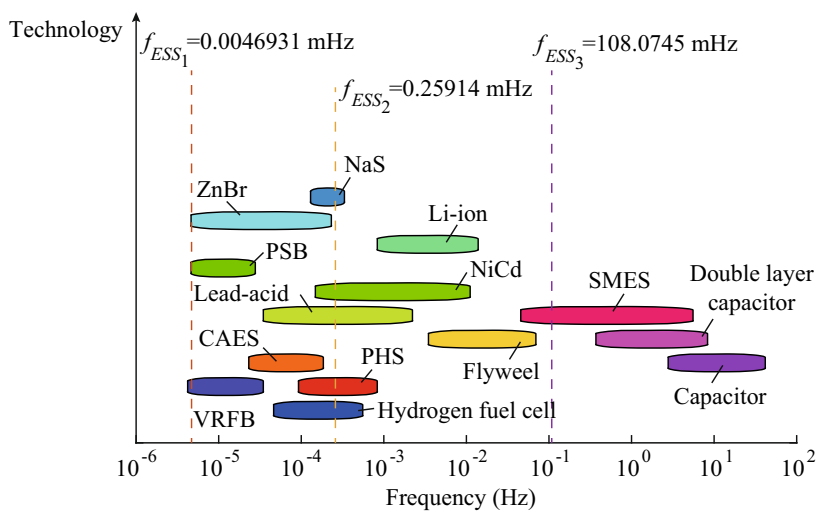

Fig. 16 Example of Selected ESS from the second Pareto-Front solutions

turbulence, it is easier to integrate successfully the produced energy in the grid. 


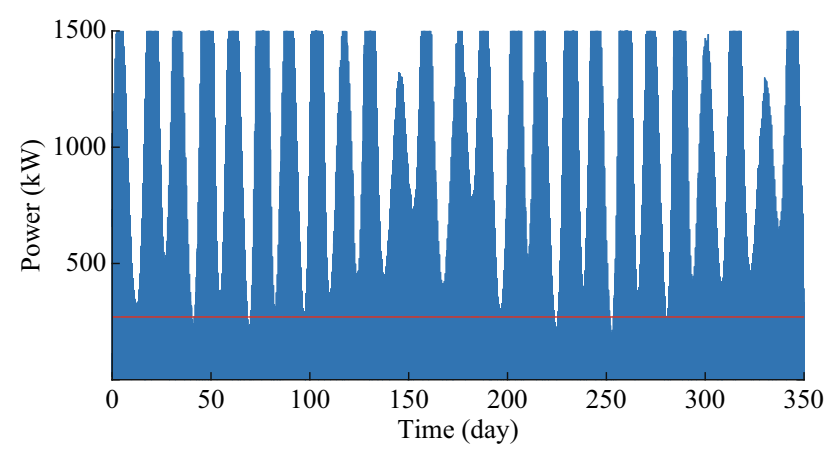

Fig. 17 Example of filtered power grid from the second Pareto-Front solutions

\section{Conclusion}

In this paper, an optimal sizing strategy for hybridized energy storage systems were presented. This approach is based on a simplified frequency energy management method. Optimal solutions are obtained using a multi-objective genetic algorithm. However, the procedure is highly time consuming, especially when using annual tidal speed data with one second as sampling time. Therefore, some simplifications were used to reduce computational time by excluding the estimation of the life span and considering a limited database of storage systems with average values of power and energy density related to average values of power capital and energy capital costs of the ESS. Nevertheless, the obtained results are very interesting and give a good idea about the optimal solutions to be considered according to their cost and performances.

Open Access This article is distributed under the terms of the Creative Commons Attribution 4.0 International License (http:// creativecommons.org/licenses/by/4.0/), which permits unrestricted use, distribution, and reproduction in any medium, provided you give appropriate credit to the original author(s) and the source, provide a link to the Creative Commons license, and indicate if changes were made.

\section{References}

[1] Eltigani D, Masri S (2015) Challenges of integrating renewable energy sources to smart grids: a review. Renew Sustain Energy Rev 52:770-770

[2] Cifor A, Denholm P, Ela E et al (2015) The policy and institutional challenges of grid integration of renewable energy in the Western United States. Utilities Policy 33:34-41

[3] Hemmati R, Saboori H (2016) Emergence of hybrid energy storage systems in renewable energy and transport applications: a review. Renew Sustain Energy Rev 65:11-23

[4] Parra D, Gillott M, Norman SA et al (2015) Optimum community energy storage system for PV energy time-shift. Appl Energy 137:576-587

[5] Friis F, Christensen TH (2016) The challenge of time shifting energy demand practices: insights from Denmark. Energy Res Soc Sci 19(Supplement C):124-133
[6] Parra D, Norman SA, Walker GS et al (2017) Optimum community energy storage for renewable energy and demand load management. Appl Energy 200:358-369

[7] Hemmati R, Saboori H (2017) Stochastic optimal battery storage sizing and scheduling in home energy management systems equipped with solar photovoltaic panels. Energy Build 152:290-300

[8] Olaszi BD, Ladanyi J (2017) Comparison of different discharge strategies of grid-connected residential PV systems with energy storage in perspective of optimal battery energy storage system sizing. Renew Sustain Energy Rev 75:710-718

[9] Schneider M, Biel K, Pfaller S et al (2015) Optimal sizing of electrical energy storage systems using inventory models. Energy Procedia 73:48-58

[10] Schneider M, Biel K, Pfaller S et al (2016) Using inventory models for sizing energy storage systems: an interdisciplinary approach. J Energy Storage 8:339-348

[11] Cardenas R, Pena R, Asher G et al (2001) Control strategies for enhanced power smoothing in wind energy systems using a flywheel driven by a vector-controlled induction machine. IEEE Trans Ind Electron 48(3):625-635

[12] Brekken TKA, Yokochi A, Jouanne AV et al (2011) Optimal energy storage sizing and control for wind power applications. IEEE Trans Sustain Energy 2(1):69-77

[13] Cimuca GO, Saudemont C, Robyns B et al (2006) Control and performance evaluation of a flywheel energy-storage system associated to a variable-speed wind generator. IEEE Trans Ind Electron 53(4):1074-1085

[14] Xiao J, Bai L, Li F et al (2014) Sizing of energy storage and diesel generators in an isolated microgrid using discrete fourier transform (DFT). IEEE Trans Sustain Energy 5(3):907-916

[15] Makarov YV, Du P, Kintner-Meyer MCW et al (2012) Sizing energy storage to accommodate high penetration of variable energy resources. IEEE Trans Sustain Energy 3(1):34-40

[16] Liu Y, Du W, Xiao L et al (2016) A method for sizing energy storage system to increase wind penetration as limited by grid frequency deviations. IEEE Trans Power Syst 31(1):729-737

[17] Tani A, Camara MB, Dakyo B (2012) Energy management in the decentralized generation systems based on renewable energy. In: Proceedings of international conference on renewable energy research and applications, Nagasaki, Japan, 11-14 November 2012, 6 pp

[18] Belouda M, Jaafar A, Sareni B et al (2016) Design methodologies for sizing a battery bank devoted to a stand-alone and electronically passive wind turbine system. Renew Sustain Energy Rev 60:144-154

[19] Sarrias-Mena R, Fernndez-Ramrez LM, GarcaVzquez CA et al (2014) Improving grid integration of wind turbines by using secondary batteries. Renew Sustain Energy Rev 34:194-207

[20] Myers L, Bahaj A (2005) Simulated electrical power potential harnessed by marine current turbine arrays in the alderney race. Renew Energy 30(11):1713-1731

[21] Elghali SEB, Balme R, Saux KL et al (2007) A simulation model for the evaluation of the electrical power potential harnessed by a marine current turbine. IEEE J Ocean Eng 32(4):786-797

[22] Zhou Z, Scuiller F, Charpentier JF et al (2013) Power smoothing control in a grid-connected marine current turbine system for compensating swell effect. IEEE Trans Sustain Energy $4(3): 816-826$

[23] Zhou Z, Scuiller F, Charpentier JF et al (2012) Grid-connected marine current generation system power smoothing control using supercapacitors. In: Proceedings of 38th annual conference on IEEE industrial electronics society, Montreal, Canada, 25-28 October 2012, 6 pp 
[24] Jaafar A, Akli CR, Sareni B et al (2009) Sizing and energy management of a hybrid locomotive based on flywheel and accumulators. IEEE Trans Veh Technol 58(8):3947-3958

[25] Akli CR, Roboam X, Sareni B et al (2007) Energy management and sizing of a hybrid locomotive. In: Proceedings of 2007 European conference on power electronics and applications, Aalborg, Denmark, 2-5 September 2007, 10 pp

[26] Christen T, Carlen MW (2000) Theory of ragone plots. J Power Sources 91(2):210-216

[27] Akli CR (2008) Conception systémique d'une locomotive hybride autonome: application à la locomotive hybride de démonstration et d'investigations en énergétique LhyDIE développée par la SNCF. Dissertation, ENSEEIHT

[28] Luo X, Wang J, Dooner M et al (2015) Overview of current development in electrical energy storage technologies and the application potential in power system operation. Appl Energy 137:511-536

[29] Elghali SEB, Benbouzid MEH, Charpentier J (2010) Modelling and control of a marine current turbine-driven doubly fed induction generator. IET Renew Power Gener 4:1-11

[30] Deb K, Pratap A, Agarwal S et al (2002) A fast and elitist multiobjective genetic algorithm: Nsga-ii. IEEE Trans Evol Comput 6(2):182-197

Seifeddine BEN ELGHALI received the B.Sc. degree in Electrical Engineering in 2005 from ENIT, Tunis, Tunisia, the M.Sc. degree in Automatic Control in 2006 from the University of Poitiers, and the $\mathrm{Ph} . \mathrm{D}$. degree in Electrical Engineering in 2009 from the University of Brest. Since 2010, he is an Associate Professor of Electrical Engineering in the University of Aix-Marseille, France. His research interests include modeling and control of renewable energy applications.
Rachid OUTBIB received his Ph.D. degree in applied mathematics in 1994 and his Habilitation à Diriger des Recherches degree in automatics control in 1998, respectively from the University of Metz and the University of Amiens (France). He was Full Professor at the University of Technology at Belfort (France), from 2003 to 2006. Since 2007, he is Full Professor at the University of Aix-Marseille. He is head of the ESCODI a team of the LSIS_lab. His research interests include nonlinear systems methods with applications to fluid power, automotive and energetic systems.

Mohamed BENBOUZID received the B.Sc. degree in Electrical Engineering from the University of Batna, Batna, Algeria, in 1990, the M.Sc. and Ph.D. degrees in Electrical and Computer Engineering from the National Polytechnic Institute of Grenoble, Grenoble, France, in 1991 and 1994, respectively, and the Habilitation à Diriger des Recherches degree from the University of Picardie "Jules Verne," Amiens, France, in 2000. After receiving the Ph.D. degree, he joined the Professional Institute of Amiens, University of Picardie "Jules Verne," where he was an Associate Professor of electrical and computer engineering. Since September 2004, he has been with the Institut Universitaire de Technologie of Brest, University of Brest, Brest, France, where he is a Professor of electrical engineering. He is also a Distinguished Professor at the Shanghai Maritime University, Shanghai, China. His research interests include analysis, design, and control of electric machines, variable-speed drives for traction, propulsion, and renewable energy applications, and fault diagnosis of electric machines. He is an IEEE Senior Member. He is the Editor-inChief of the Inrernational Journal on Energy Conversion (IRECON). $\mathrm{He}$ is also an Associate Editor of the IEEE Transactions on Energy Conversion, the IEEE Transactions on Industrial Electronics, the IEEE Transactions on Sustainable Energy, the IEEE Transactions on Vehicular Technology. $\mathrm{He}$ is a Subject Editor for the IET Renewable Power Generation. 